\title{
Dynamics of multishell vortex structures in mesoscopic superconducting Corbino disks
}

\author{
N. S. Lin, ${ }^{1}$ V. R. Misko, ${ }^{1}$ and F. M. Peeters ${ }^{1,2}$ \\ ${ }^{1}$ Department of Physics, University of Antwerpen, Groenenborgerlaan 171, B-2020 Antwerpen, Belgium \\ ${ }^{2}$ Departamento de Física, Universidade Federal do Ceará, 60455-900 Fortaleza, CE, Brazil \\ (Received 7 November 2009; revised manuscript received 2 February 2010; published 2 April 2010)
}

\begin{abstract}
We study the dynamics of vortex shells in mesoscopic superconducting Corbino disks, where vortices form shells as recently observed in micrometer-sized Nb disks. Due to the interplay between the vortex-vortex interaction, the gradient Lorentz force and the (in)commensurability between the numbers of vortices in shells, the process of angular melting of vortex-shell configurations becomes complex. Angular melting can start either from the center of the disk (where the shear stress is maximum) or from its boundary (where the shear stress is minimum) depending on the specific vortex configuration. Furthermore, we found that two kinds of defects can exist in such vortex-shell structures: intrashell and intershell defects. An intrashell defect may lead to an inverse dynamic behavior, i.e., one of the vortex shells under a stronger driving force can rotate slower than the adjacent shell that is driven by a weaker Lorentz force. An intershell defect always locks more than two shells until the gradient of the Lorentz force becomes large enough to break the rigid-body rotation of the locked shells. Such a lock-unlock process leads to hysteresis in the angular velocities of the shells.
\end{abstract}

DOI: $10.1103 /$ PhysRevB.81.134504

PACS number(s): 74.25.Uv, 74.78.Na, 68.35.Af

\section{INTRODUCTION}

The dynamics of vortex matter in mesoscopic and nanostructured superconductors has attracted an increasing interest of researchers studying the fundamental properties of vortex matter and its practical applications in superconducting devices. Over the last decade, the dynamics of vortices in superconducting disks has been actively studied by using a Corbino geometry. ${ }^{1-8}$ In a Corbino geometry (see Fig. 1), a superconducting disk is placed in an external perpendicular magnetic field $H_{0}$ with a radial driving current $I$, which is injected at the center of the disk and removed from its edge. The density of the current $j$ decays as $1 / \rho$ along the radius, ${ }^{4-8}$ so the driving force (i.e., the Lorentz force) $f^{d}$ that acts on a vortex near the center of the disk is larger than that close to the edge. This natural gradient of the driving force allows a direct examination of the elastic moduli of the vortex lattice, e.g., the shear modulus.

In an infinite superconductor and in thin films, vortices form a triangular Abrikosov lattice ${ }^{9}$ as a consequence of the isotropic repulsive vortex-vortex interaction. Therefore in large disks most of vortices are packed in a triangular lattice except those near the interface. The transition from elastic (i.e., vortex lattice under elastic deformations) to plastic (i.e., vortex liquid) vortex motion in Corbino disks has been investigated in Refs. 2, 6, and 8. Two sharp jumps of the global resistance at two critical current values in large disks have been found. ${ }^{6}$ The system transits from rigid-body motion to plastic flow motion and proceeds from plastic motion to laminar motion at those two jumps, respectively.

Due to the interaction between the vortices and the edge, the configuration of vortices in mesoscopic disks experiences a transition from the Abrikosov lattice to vortex shells with decreasing size of the system. ${ }^{10}$ Vortices obey specific rules for filling shells in small disks, which have been found in numerical simulations ${ }^{11,12}$ and were recently observed in experiments by using the Bitter decoration technique. ${ }^{13,14}$ The dynamics of vortex shells in small mesoscopic Corbino disks, having only two or three shells, was investigated in Ref. 7. By applying an increasing external current $I_{0}$ to such a system, the vortex shells unlock at a critical value of the current $I_{c}$ and start to rotate with different angular velocities (which is called "angular melting" "7,15-19) while for $I_{0}<I_{c}$ all


FIG. 1. (Color online) (a) Schematic plot of a Corbino disk: a current is injected at the center of the disk and flows radially to the edge in the presence of an external perpendicular magnetic field $H_{0}$. (b) The experimental setup of a macroscopic Corbino disk (after Ref. 4). The vortex is driven by the gradient Lorentz force due to the inhomogeneous density of the current along the radius. 
the vortices rotate as a rigid body. With increasing magnetic field, a "structural transition" was revealed when a vortex jumped from the outer shell to the inner shell, similar to the Coster-Kronig transition in hollow atoms. ${ }^{7}$ A two-step angular melting was found in a three-shell system with increasing temperature for driving current below the depinning threshold, similar to what was found in experiments. ${ }^{4,5}$

In the present study, we are interested in large mesoscopic disks that contain several vortex shells, i.e., the intermediate case between small mesoscopic disks and macroscopic disks where vortices form a lattice. In view of the interplay between the vortex-vortex interaction and the confinement, our findings will lead to a better understanding of the rich dynamics of multishell systems. Note that in our previous work, ${ }^{18}$ we demonstrated the possibility of unusual ("unconventional") dynamics and melting of shells in mesoscopic Corbino disks. Here, we analyze the free energy and the statistics of different vortex states, discuss the mechanism of intershell "friction" in commensurate and incommensurate shells, and systematically investigate the dynamics of vortex shells by considering many different vortex configurations (VC). We classify different types of defects and analyze the propagation of a "compression-decompression wave," induced by defects that results in "unconventional" dynamics of vortex shells.

The paper is organized as follows. We describe the simulation method in Sec. II. Then, in order to find the ground state and examine the stability of different configuration states, the free energy and the probability of different configurations will be analyzed in Sec. III. After that, we will present in Sec. IV the different dynamical processes that are responsible for angular melting in mesoscopic Corbino disks. The first part of this section will show two different scenarios of angular melting for different configurations: the angular melting starting either at the center or near the edge. In addition, the dynamics of vortex shells in two specific cases will be discussed in Sec. IV. In such a way, we will reveal two kinds of defects which respectively induce: (i) an inverse dynamical behavior, i.e., an inner shell moving slower than its outer neighbor and (ii) a hysteresis effect of the angular velocity. Finally, our conclusions will be given in Sec. V.

\section{SIMULATION}

In order to study the dynamics of vortices driven by the Lorentz force that is induced by the external current, we perform Langevin-type molecular-dynamics (MD) simulations and numerically integrate the overdamped equations of motion: $7,11,12,14,18,20$

$$
\eta \frac{d \boldsymbol{r}_{i}}{d t}=\boldsymbol{f}_{i}
$$

with

$$
\boldsymbol{f}_{i}=\boldsymbol{f}_{i}^{v v}+\boldsymbol{f}_{i}^{T}+\boldsymbol{f}_{i}^{d}+\boldsymbol{f}_{i}^{s}
$$

where $\eta$ is the viscosity coefficient which is set here to unity, and $\boldsymbol{f}_{i}^{v v}, \boldsymbol{f}_{i}^{T}, \boldsymbol{f}_{i}^{d}$, and $\boldsymbol{f}_{i}^{s}$ are the vortex-vortex interaction force, the thermal force, the driving force (Lorentz force), and the force due to the shielding currents and the edge, respectively. ${ }^{7,12,14,20}$ Note that the disorder (i.e., due to the intrinsic pinning) results in the overdamped regime of vortex motion. At the same time, disks are assumed to be free of artificial inclusions or holes (i.e., artificial pinning centra) and thus the vortex-pin interaction has not been explicitly included in the equation of motion, Eq. (1) (cp. Refs. 20-22). The thermal force $\boldsymbol{f}_{i}^{T}$ obeys the following conditions:

$$
\left\langle\boldsymbol{f}_{i}^{T}(t)\right\rangle=0
$$

and

$$
\left\langle\boldsymbol{f}_{i}^{T}(t) \boldsymbol{f}_{j}^{T}\left(t^{\prime}\right)\right\rangle=2 \eta k_{B} T \delta_{i j} \delta\left(t-t^{\prime}\right) .
$$

An applied current flows from the center of the disk to the edge and thus the density of the current is inversely proportional to the radius $\rho$,

$$
J(\rho)=\frac{I}{2 \pi \rho d},
$$

where $d$ is the thickness of the superconducting disk. Therefore, the Lorentz force acting on the outer vortex, i.e., near the boundary, is much weaker than that acting on the inner one, i.e., close to the center. To avoid divergency of the current density at the center, we introduced a cutoff radius $\rho_{0}$ $=10^{-3} R$. Inside of the cutoff radius, the density of the current was assumed to be constant: $J_{0}=J\left(\rho_{0}\right)$. The driving force (per unit length) due to the radial current is

$$
f_{i}^{d}=\frac{\Phi_{0} I}{2 \pi \rho_{i} d} \hat{\theta}=\frac{f_{0} I_{0}}{r_{i}} \hat{\theta},
$$

where $r_{i}=\rho_{i} / R, \hat{\theta}$ is the unit vector in the azimuthal direction in the disk plane, $f_{0}=\Phi_{0}^{2} /\left(2 \pi \mu_{0} R \lambda^{2}\right)=4 \pi \mu_{0} \xi^{2} H_{c}^{2} / R$ is the unit of force, and $I_{0}=\mu_{0} \lambda^{2} I /\left(\Phi_{0} d\right)=\mu_{0} \Lambda I / \Phi_{0}$ is the dimensionless driving current. Here $R$ is the radius of the disk. We treat vortices as massless classical particles (CPs) and assume that the motion of the vortices does not influence the distribution of the applied current density. Therefore, the driving force is always applied in the azimuthal direction and thus no Hall effect is taken into account.

In a thin superconductor such that $\Lambda=\lambda^{2} / d \gg R \gg \xi \gg d$, the vortex-vortex interaction force $f_{i}^{v v}$ is modeled by ${ }^{7,11,23}$

$$
\boldsymbol{f}_{i}^{v v}=f_{0} \sum_{i, k}^{L}\left(\frac{\boldsymbol{r}_{i}-\boldsymbol{r}_{k}}{\left|\boldsymbol{r}_{i}-\boldsymbol{r}_{k}\right|^{2}}-r_{k}^{2} \frac{r_{k}^{2} \boldsymbol{r}_{i}-\boldsymbol{r}_{k}}{\left|r_{k}^{2} \boldsymbol{r}_{i}-\boldsymbol{r}_{k}\right|^{2}}\right)
$$

and the force due to the vortex interaction with the shielding currents and the edge $f_{i}^{s}$ is ${ }^{7,11,23}$

$$
\boldsymbol{f}_{i}^{s}=f_{0}\left(\frac{1}{1-r_{i}^{2}}-h\right) \boldsymbol{r}_{i}
$$

with the dimensionless applied magnetic field $h$ $=\pi R^{2} \mu_{0} H_{0} / \Phi_{0}=\left(H_{0} / 2 H_{c 2}\right)(R / \xi)^{2}$.

In our simulations, we first set $T>0$, when no current is applied, and then gradually decrease temperature to let the system relax to the ground state. ${ }^{24-26}$ In this way, we simulated the stimulated annealing process similar to that in annealing experiments. ${ }^{27,28}$ Then, we set $T=0$ and apply the current to study the dynamics of the system of vortices. 


\section{VORTEX CONFIGURATIONS AND THEIR STABILITY}

The system of many interacting vortices in a disk has many local minima corresponding to metastable (MS) states. We analyze the stability of different vortex configurations by calculating their free energy and probability of appearance.

For vorticity (i.e., the number of vortices in a disk) $L$ $>5$, the ground-state (GS) and metastable configurations of vortices in mesoscopic disks have been found both in simulations ${ }^{11,12}$ and in experiments. ${ }^{13}$ To find the ground state at different magnetic field $H_{0}$, we calculated the improved London free energy, ${ }^{10}$

$$
\mathcal{F}=\mathcal{F}_{L}+\mathcal{F}_{\text {core }}
$$

with

$$
\begin{gathered}
\mathcal{F}_{L}=\sum_{i=1}^{L}\left(\epsilon_{i}^{\text {self }}+\epsilon_{i}^{\text {shield }}+\sum_{j=1}^{i-1} \epsilon_{i j}\right)+\epsilon^{\text {core }}+\epsilon^{\text {field }}, \\
\mathcal{F}_{\text {core }} \approx-1+L \frac{3}{R^{2}} .
\end{gathered}
$$

This London energy, Eq. (9), contains two parts. One is the usual London energy and the other one is related to the vortex cores contribution. When $\kappa \gg 1$, vortex cores do not overlap $^{29}$ and $\Lambda=\lambda^{2} / d \gg \xi \gg d$, then we obtain the usual London energy $\mathcal{F}_{L}$. However, the usual London limit neglects the spatial variation in $\left|\Psi^{2}\right|$. By introducing a function (which varies from 0 to 1 within the range $\left.\left|\rho-\rho_{i}\right| \sim \xi\right)$ to approximate the size of the vortex core, the contribution of the vortex cores have been taken into account. Assuming the vortex core has radius $\sqrt{2} \xi$, we obtain the contribution $\mathcal{F}_{\text {core }}$. Thus the "improved London energy" is the sum of these two contributions, which provide an agreement with the GinzburgLandau result as was shown in Ref. 10.

Figure 2 shows the free energy as a function of magnetic field in a disk with $R=50 \xi$. Note that the difference in the free energy between two states with different $L$ is much larger than that between two different configurations with the same $L$ [see the inset of Fig. 2(c)].

Analyzing the energies of different configurations with the same $L$, we find the range of magnetic field values in which the ground-state-energy configuration remains the same. For instance, the configuration (1-6-12-19-25) with $L$ $=62$ has the lowest energy for $75.9<h<76.7$. Compared to the states of charged CPs, the ground states [column "ground state (VC)"] and the metastable states [column "metastable (VC)" with different $L$ in disks with $R=50 \xi$ are listed in Table I.

In order to find the probabilities of appearance of different metastable configurations, we performed number (usually 100) simulations for the same $L$ starting from different random initial vortex configurations. Although it may not cover all possible configurations, the statistic of 100 simulations gives a rather representative sampling these stable configurations. In this way, the "most probable" vortex configuration (i.e., the configuration with the highest probability of appearance) for a certain $L$ is found. The probabilities are shown in Fig. 3 for different vortex states, for $L=63,92$, and 93. We


FIG. 2. (Color online) The energies of different vortex configurations with vorticities: (a) $L=33-39$; (b) $L=59-64$; and (c) $L$ $=86-94$. The ground state is denoted by the letter " $G$ " if there are several different configurations with the same $L$ in (c) and these configurations are distinguished by using Greek letters " $\alpha$ " $(i=1)$, “ $\beta$ " $(i=2)$, " $\gamma$ " $(i=3), " \delta$ " $(i=4)$, and " $\varepsilon$ " $(i=5)$, which denote the $i$ th shell with $6 i+1$ vortices (i.e., shell $S_{6 i+1}^{i}$ ) while the others have $6 i$ vortices (i.e., shell $S_{6 i}^{i}$ ), respectively. Here $S_{j}^{i}$ is introduced to denote the $i$ th shell which contains $j$ vortices. The difference in energy between two configurations with different vorticities $L$ is much larger than that between two configurations with the same $L$ as is illustrated by the inset of (c). The regions of magnetic field where the ground-state-energy configuration is provided by the same $L$ are separated by vertical black dotted lines.

find that the most probable configuration is not always the lowest-energy state for given values of $L$. For instance, for $L=92$, the state (1-6-12-19-24-30) is the most probable state [Fig. 3(b)] with $46 \%$ appearance while the ground state (1$6-12-18-24-31)$ is found in only $15 \%$ of the cases. Previously 
TABLE I. The GS and MS VC in a disk with $R=50 \xi$ and the ground state and metastable state of CPs (Refs. 15 and 30). A typical value of magnetic field $h$ corresponding to the ground state is also given.

\begin{tabular}{|c|c|c|c|c|c|c|c|}
\hline \multirow{2}{*}{$\frac{L}{33}$} & \multirow{2}{*}{$\frac{h}{45}$} & \multirow{2}{*}{$\frac{\mathrm{GS}(\mathrm{VC})}{5-11-17}$} & \multicolumn{2}{|c|}{ MS (VC) } & \multirow{2}{*}{$\frac{G S(C P s)^{a}}{1-6-11-15}$} & \multicolumn{2}{|c|}{$\mathrm{MS}(\mathrm{CPs})^{\mathrm{a}}$} \\
\hline & & & $1-5-11-16$ & & & $1-5-11-16$ & $1-6-10-16$ \\
\hline \multirow[t]{2}{*}{34} & 46 & $1-5-11-17$ & $5-12-17$ & $6-11-17$ & $1-6-12-15$ & $1-6-11-16$ & $1-7-11-15$ \\
\hline & & & & & & & $1-5-11-17$ \\
\hline \multirow[t]{2}{*}{35} & 47 & $1-5-12-17$ & $1-5-11-18$ & & $1-6-12-16$ & $1-6-11-17$ & $1-7-11-16$ \\
\hline & & & & & & & $1-6-13-15$ \\
\hline \multirow[t]{2}{*}{36} & 48 & $1-6-12-17$ & $1-6-11-18$ & $1-5-12-18$ & $1-6-12-17$ & $1-7-12-16$ & $1-6-13-16$ \\
\hline & & & & & & & $1-7-13-15$ \\
\hline 37 & 49 & $1-6-12-18$ & & & $1-7-12-17$ & $1-7-13-16$ & $1-6-13-17$ \\
\hline \multirow[t]{2}{*}{38} & 50 & $1-6-12-19$ & $1-6-13-18$ & & $1-7-13-17$ & $2-7-13-16$ & $2-7-12-17$ \\
\hline & & & & & & & $1-7-14-16$ \\
\hline \multirow[t]{2}{*}{39} & 51 & $1-6-13-19$ & $1-7-12-19$ & & $2-7-13-17$ & $1-7-13-18$ & $1-7-14-17$ \\
\hline & & & & & & & $2-7-14-16$ \\
\hline 61 & 75 & $1-6-12-18-24$ & $1-7-12-18-23$ & & & & \\
\hline 62 & 76 & $1-6-12-19-24$ & $1-6-13-18-24$ & $1-7-12-18-24$ & & & \\
\hline 63 & 77 & $1-6-12-19-25$ & $1-6-13-19-24$ & $1-7-13-18-24$ & & & \\
\hline 64 & 78 & $1-7-13-19-24$ & $1-7-13-18-25$ & & & & \\
\hline 90 & 106 & $1-6-12-18-24-29$ & & & & & \\
\hline 91 & 107 & $1-6-12-18-24-30$ & & & & & \\
\hline \multirow[t]{2}{*}{92} & 108 & $1-6-12-18-24-31$ & $1-6-12-18-25-30$ & $1-6-12-19-24-30$ & & & \\
\hline & & & $1-6-13-18-24-30$ & $1-7-12-18-24-30$ & & & \\
\hline \multirow[t]{2}{*}{93} & 109 & $1-6-12-18-25-31$ & $1-6-12-19-25-30$ & $1-6-13-19-24-30$ & & & \\
\hline & & & $1-7-13-18-24-30$ & $1-6-13-18-24-31$ & & & \\
\hline 94 & 110 & $1-7-13-19-24-30$ & $1-6-13-19-25-30$ & $1-6-13-19-24-31$ & & & \\
\hline
\end{tabular}

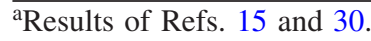

similar results were found in the case of small disks ${ }^{12}$ which was explained as due to the fact that the ground state is reached through narrower minima in configuration space while the most probable state corresponded to a broader local minimum that is separated from the ground state by an energy barrier. Note that, in principle, when the annealing is slow enough and the number of initial random realizations is large enough such that the whole configurational space (i.e., all possible vortex configurations) is covered, the "lowestenergy" and the most probable states should coincide. However, in reality, this would require very extensive and thus very slow simulation runs for finding just initial vortex distributions. The fact that the most probable state does not coincide with the lowest-energy state means that this state is a metastable state with an energy very close to the energy of the ground-state configuration. It is important that this way of finding the initial vortex configuration has a direct experimental realization when a hundred (or 200-300) identical disks are measured at the same conditions and then the statistics of different states is analyzed (see Refs. 13 and 14). Thus, by finding the most probable state we simulate the experimental conditions. ${ }^{13,14}$ We have found that some of these most probable states exhibit interesting dynamical behavior which will be discussed in the next section.

\section{ANGULAR MELTING: DIFFERENT SCENARIOS}

We define the average velocity of all vortices in the $i$ th shell as the "angular velocity of the $i$ th shell," $\omega_{i}$. The angular velocity of each shell $\left\{\omega_{i} \mid i=1,2, \ldots\right\}$ is calculated as a function of the applied current $I_{0}$, i.e., $\omega\left(I_{0}\right)$ curves will be plotted.

Because the Lorentz force, i.e., the driving force $f^{d}$, is maximum at the center of the disk and reduces as $1 / \rho$ along the radius, one can expect that inner shells unlock first, i.e., angular melting starts at the center and propagates toward the periphery. This behavior is indeed realized in macroscopic disks. However, in mesoscopic disks with a shell structure the process of melting is more complex due to commensurability/incommensurability between the shells resulting in different scenarios of angular melting. We discuss these different scenarios below.

\section{A. Commensurability/incommensurability and angular melting}

The shells are called commensurate when the numbers of vortices in those shells are commensurate, i.e., they have a common divisor. ${ }^{31}$ Commensurate vortex shells usually contains $6 i$ vortices, where $i$ is an integer. These shells are strongly locked because of a relatively larger energy barrier due to the interaction with the vortices in adjacent shell(s) 

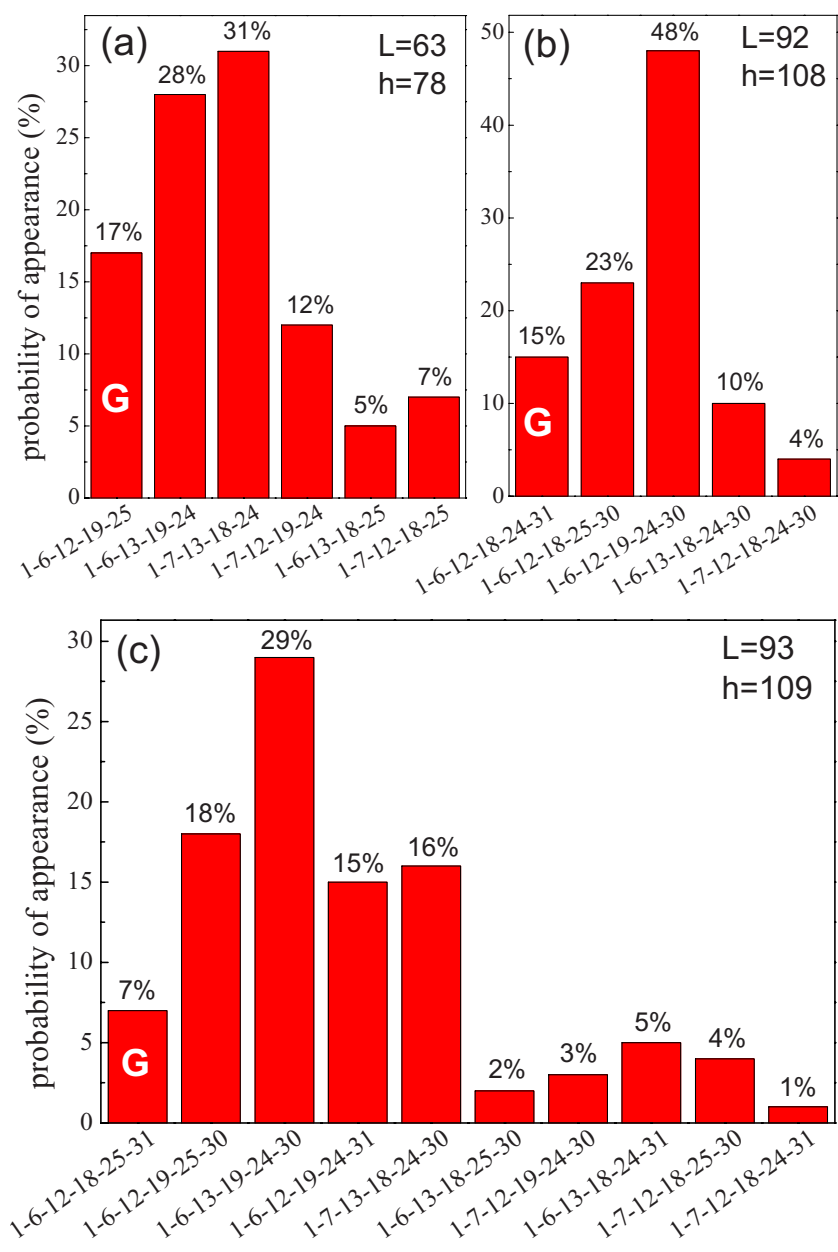

FIG. 3. (Color online) The probability of the appearance of different vortex configurations with vorticity (a) $L=63$, (b) $L=92$, and (c) $L=93$. The most probable state is not always the ground state (denoted by G). For instance, for $L=63$, the ground state is (1-612-19-25) with probability $17 \%$, which is smaller than the probabilities of states (1-6-13-19-24) and (1-7-13-18-24).

resulting in a larger intershell friction and thus a larger shear stress is required to unlock them than in the case of incommensurate shells which can easily slide with respect to each other (see Fig. 4). To illustrate the effect of commensurate and incommensurate shells, we consider here disks with vorticity $L=38$, which represent typical configurations with small vorticity having both commensurate and incommensurate shells. We find three (meta)stable configurations for $L$ $=38$ which are (1-7-12-18), (1-6-13-18), and (1-6-12-19). We introduced $S_{j}^{i}$ to denote the $i$ th shell which contains $j$ vortices. For example, shell $S_{7}^{1}$ of the configuration (1-7-12-18), shell $S_{13}^{2}$ of the configuration (1-6-13-18), and shell $S_{19}^{3}$ of the configuration (1-6-12-19) are incommensurate shells. By studying the angular velocities of shells, we find that incommensurate shells split off at lower driving force (shown in Fig. 5), as one intuitively expects.

Shell $S_{7}^{1}$ [Fig. 5(a)] splits off first due to both a larger gradient of the Lorentz force and a weaker friction (i.e., a smaller energy barrier) with the incommensurate adjacent shell $S_{12}^{2}$. However, while shell $S_{6}^{1}$ [Fig. 5(c)] experiences a larger gradient of the Lorentz force, it also has a stronger
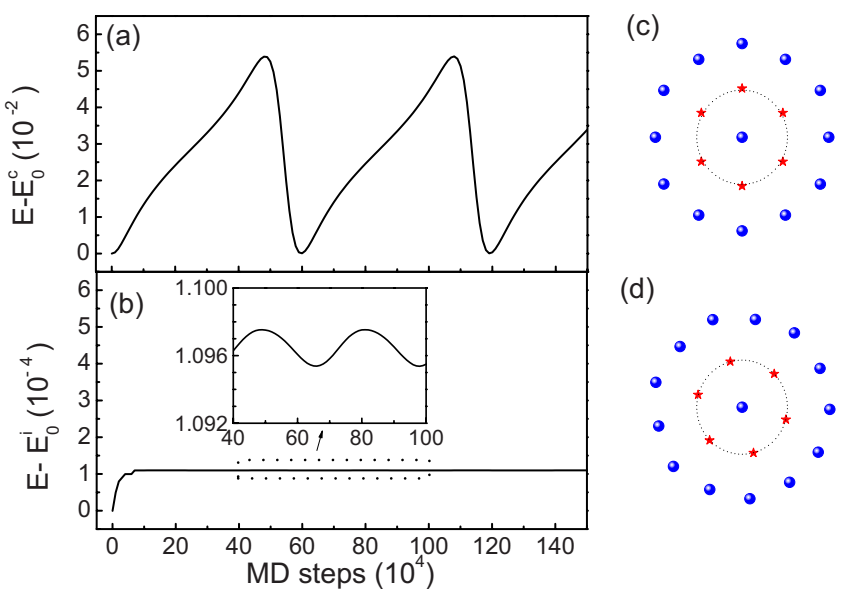

(d)

FIG. 4. (Color online) The energy barriers produced by vortices of the outer shell: (a) in commensurate case and (b) in incommensurate case. The configurations with two commensurate [i.e., (1-612)] and incommensurate shells [i.e., (1-6-13)] are shown in (c) and (d), respectively. All vortices of the inner shell (stars) rotate with respect to the outer shell (balls) due to the driving force. The interaction energy between the inner shell and the outer shell $E$ is calculated for both cases. Here $E_{0}^{c}\left(E_{0}^{i}\right)$ is the interaction energy of the ground state in the commensurate (incommensurate) case. The energy barrier in (a) is much larger than that in (b); notice also the factor $10^{2}$ difference in scale. Therefore, when the shells are commensurate, the inner shell must overcome a larger friction (due to the energy barriers) to rotate individually.

friction due to the commensurate adjacent shell $S_{12}^{2}$. Therefore, there is a competition between the gradient driving force and the commensurability effect (which determines friction between adjacent shells). Our calculations show that often commensurability plays a more important role than the local gradient force, resulting in an unusual (unconventional) dynamical process. Angular melting can occur first in regions of weaker shear stress ${ }^{18}$ because of a relatively weaker friction. The commensurability effects in multishell vortex structures display themselves in a more complex way than in the case of just two (in)commensurate shells (chains). This can be illustrated by the vortex configuration shown in the inset of Fig. 5(b). Parts of the vortices of the incommensurate shell $S_{13}^{2}$ adjust themselves to the commensurate core and thus this shell actually contains a hexagonlike part which is characterized by a relatively stronger friction with adjacent shell(s). As a result, the local shear modulus of that incommensurate shell is inhomogeneous and turns out to be angle dependent. In this case, the outer commensurate shell(s) could be locked to the core via the hexagonlike part of the incommensurate shell [as, e.g., shell $S_{19}^{3}$ for $L=62$ in Fig. 6(a); see also Ref. 18] and thus rotates with the same angular velocity as the core until the current reaches a critical value and unlocks the shells. Therefore, the effect of commensurability can even involve remote shells. This makes the process of angular melting in multishell vortex structures with incommensurate shells more complex.

Commensurate shells which contain $6 i$ ( $i$ is a positive integer) vortices tend to form locally a hexagonal arrangement of vortices similar to a triangular Abrikosov lattice. On the other hand, due to the circular confinement the outermost 

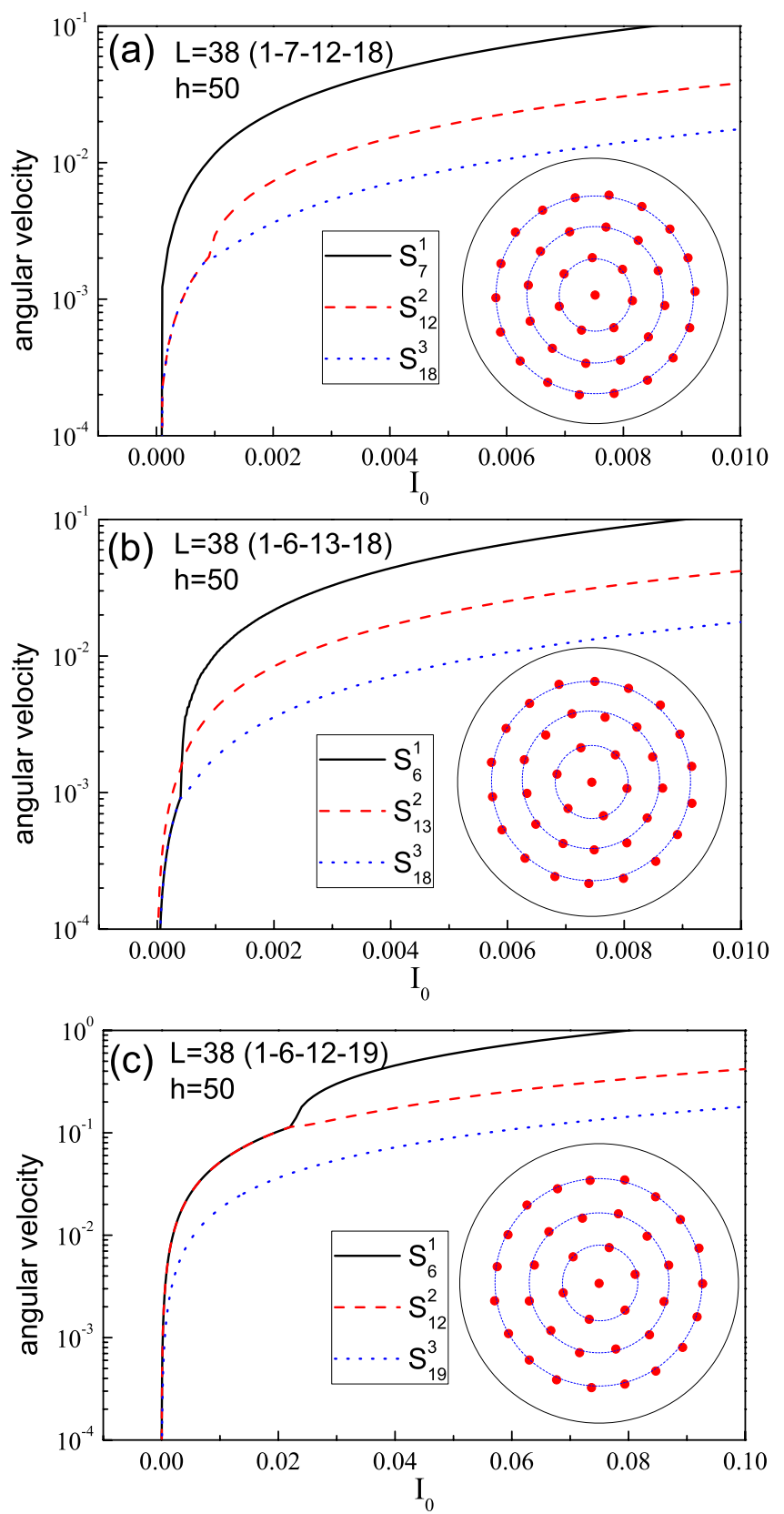

FIG. 5. (Color online) The angular velocities of shells in different configurations with $L=38$ : (a) (1-7-12-18), (b) (1-6-13-18), and (c) (1-6-12-19). The angular melting first occurs in the incommensurate shell due to a weaker friction. Different scenarios of angular melting, i.e., the first unlocking of different shell occurs in: (a) the innermost shell $S_{7}^{1}$, (b) the middle shell $S_{13}^{2}$, or (c) the outermost shell $S_{19}^{3}$.

shell is forced into a shape close to a circle. There is a transition region from the triangular Abrikosov lattice at the center to the circle shell close to the boundary. ${ }^{32}$ It is clear that two adjacent circlelike shells (or out of those shells at least one is circlelike) can slide much easier with respect to each other than two adjacent shells that are arranged in a hexagonlike configuration. This explains the above effect of unusual angular melting starting near the boundary and propagating toward the center with increasing applied current. (a)
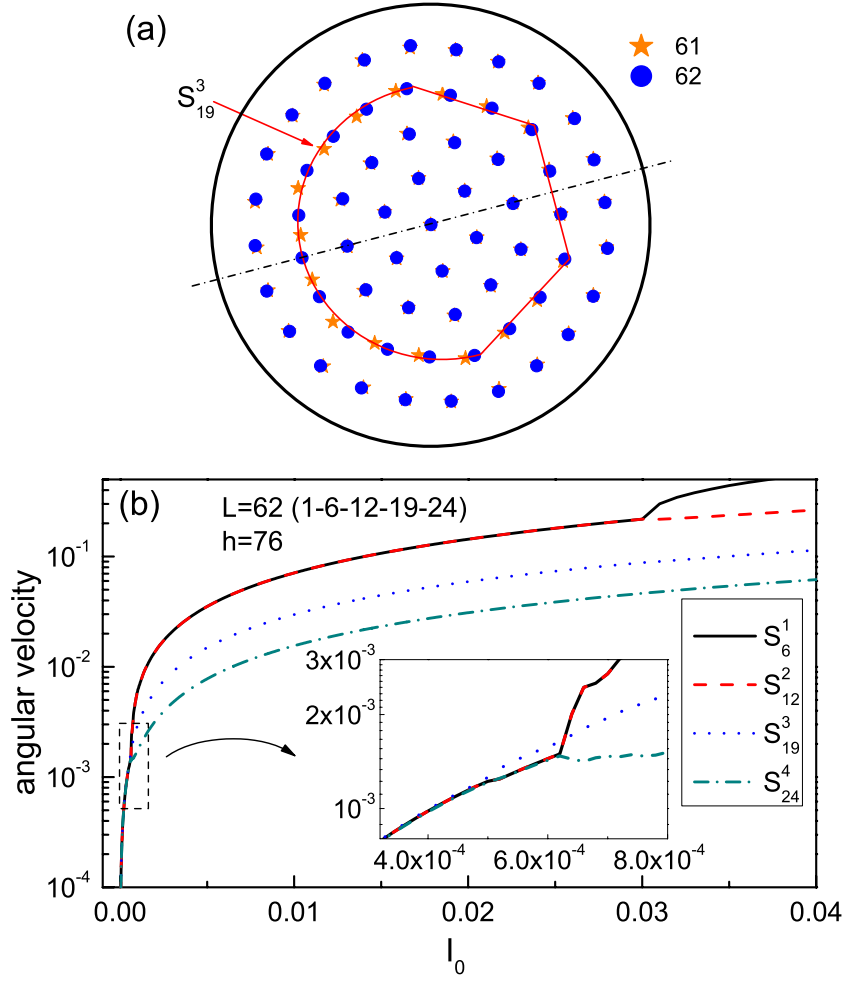

FIG. 6. (Color online) (a) Comparison between the configurations $L=61$ (1-6-12-18-24) and $L=62$ (1-6-12-19-24); (b) the angular velocities of shells for magnetic field $h=78$ with $L=62$ (1-6-1219-24); (c) the magnified image of (b). Because of an extra vortex in shell $S_{19}^{3}$ (i.e., shell $S_{19}^{3}$ is incommensurate) the friction between the adjacent shells $S_{12}^{2}$ and $S_{24}^{4}$ is small. Therefore, shell $S_{19}^{3}$ can easily slide between the adjacent shells.

\section{B. Intrashell defects and inverse angular velocities}

Due to the radially decreasing driving force, outer shells are expected to rotate slower than inner ones, except in the rigid-body regime where all shells rotate together with the same angular velocity. However, we found that for certain configurations and in specific ranges of current that the angular velocity becomes inverse, i.e., a $i$ th shell with radius $r_{i}$ rotates with a larger angular velocity than the inner adjacent shell $i-1$ with radius $r_{i-1}<r_{i}$. For example, in case of $L$ $=38$ (1-6-13-18) shell $S_{13}^{2}$ has the largest angular velocity when it unlocks while shells $S_{6}^{1}$ and $S_{18}^{3}$ still rotate together [shown in Fig. 5(b)]. Such unusual dynamical behavior is caused by the mismatch between shells along the azimuthal direction, in particular, when there are incommensurate shells (usually containing $6 i+1$ vortices) between two commensurate shells and we will call this an "intrashell defect."

As compared to the configuration where all shells are commensurate [i.e., the magic-number configuration (MNC) (Refs. 15, 31, and 33], an intrashell defect breaks up the sixfold symmetry of the shell and makes the part of the shell with defect "circlelike" [e.g., the left part of shell $S_{19}^{3}$ in Fig. 6(a)]. The additional vortex shifts other vortices of the shell from their stable trianglelike-lattice positions. Figure 6(a) shows the difference between $L=62$ (1-6-12-19-24) and $L$ $=61$ (1-6-12-18-24), i.e., configurations with and without defect, correspondingly. The angular velocity of each shell is 
shown in Fig. 6(b). Because of the incommensurability, shell $S_{19}^{3}$ splits off first and rotates "individually" [shown in Fig. 6(c)] while all other shells rotate with the same angular velocity. With increasing current, the commensurate shells start to unlock one by one. Commensurability can still explain the above behavior: the friction between incommensurate shell $S_{19}^{1}$ and the adjacent shells is weaker than that between other shells, and shell $S_{19}^{1}$ can relatively easily slide with respect to the other shells.

For completeness we also calculated the angular velocities for disks which contain two or more defect shells, e.g., $L=63$ (1-6-12-19-25) and $L=93$ (1-6-12-19-25-30) which are shown in Fig. 7. Analyzing this unusual dynamical behavior, we concluded that it cannot be explained only by commensurability. Therefore, a two-step angular-melting process has to be invoked in order to explain the inverse angular velocity behavior for the transition from rigid-body rotation $(v \sim \rho)$ to individual rotation of shells $(v \sim 1 / \rho)$. Hence, we define three regimes of vortex dynamics.

(I) Rigid-body motion. When the current is small, the gradient of the driving force is too small to overcome friction (due to the vortex-vortex interaction). All vortices rotate together as a rigid body.

(II) Compression-decompression motion. For a larger applied current, incommensurate shells slide with respect to the other shells. On one hand, the local shear modulus of the circlelike part of the defect shell is too small to keep the outer shell(s) rotating together with the inner shells. On the other hand, the gradient of the driving force is not large enough to overcome the friction between the hexagonlike part of the defect shell and its adjacent commensurate shell. In this case, the incommensurate (defect) shell is partly angular melted. The vortices of the melted part participate in an inhomogeneous slow motion, which releases a part of the vortices in that shell from the circlelike positions to their triangular-lattice positions and pushes another part of vortices away from their lattice positions. Such kind of compression-decompression motion (see Fig. 8) modifies the local density of vortices in the defect shell and finally results in a longitudinal wavelike propagation in the defect shell.

(III) Laminar motion. The shells finally acquire "individual" velocities over a certain value of current when the Lorentz force can overcome the friction. It means that the vortex lattice is totally angular melted. Note that the shells still remain well distinguished, i.e., vortices do not jump from one shell to the others. The latter would mean the onset of radial melting. ${ }^{7,15-17}$

The above regimes, except the second regime, have been discussed in previous sections. Inversion of angular velocities only occurs when the shells display compressiondecompression motion. For instance, we consider the configuration (1-6-12-18-25-31) for $L=93$. Shell $S_{25}^{4}$ and shell $S_{31}^{5}$ split off first as expected (shown in Fig. 9) because of incommensurability. However, shell $S_{25}^{4}$ has the smallest angular velocity until the current reaches the value $I_{c}^{*}$. The motion of shell $S_{25}^{4}$ and shell $S_{31}^{5}$ is considered as the combination of the rigid-body motion with the core (i.e., the inner shells forming a rigid $\mathrm{MNC}$ ) and the relative slow motion with respect to the core. The velocities of shells $S_{25}^{4}$ and $S_{31}^{5}$ can be expressed by the following formula:
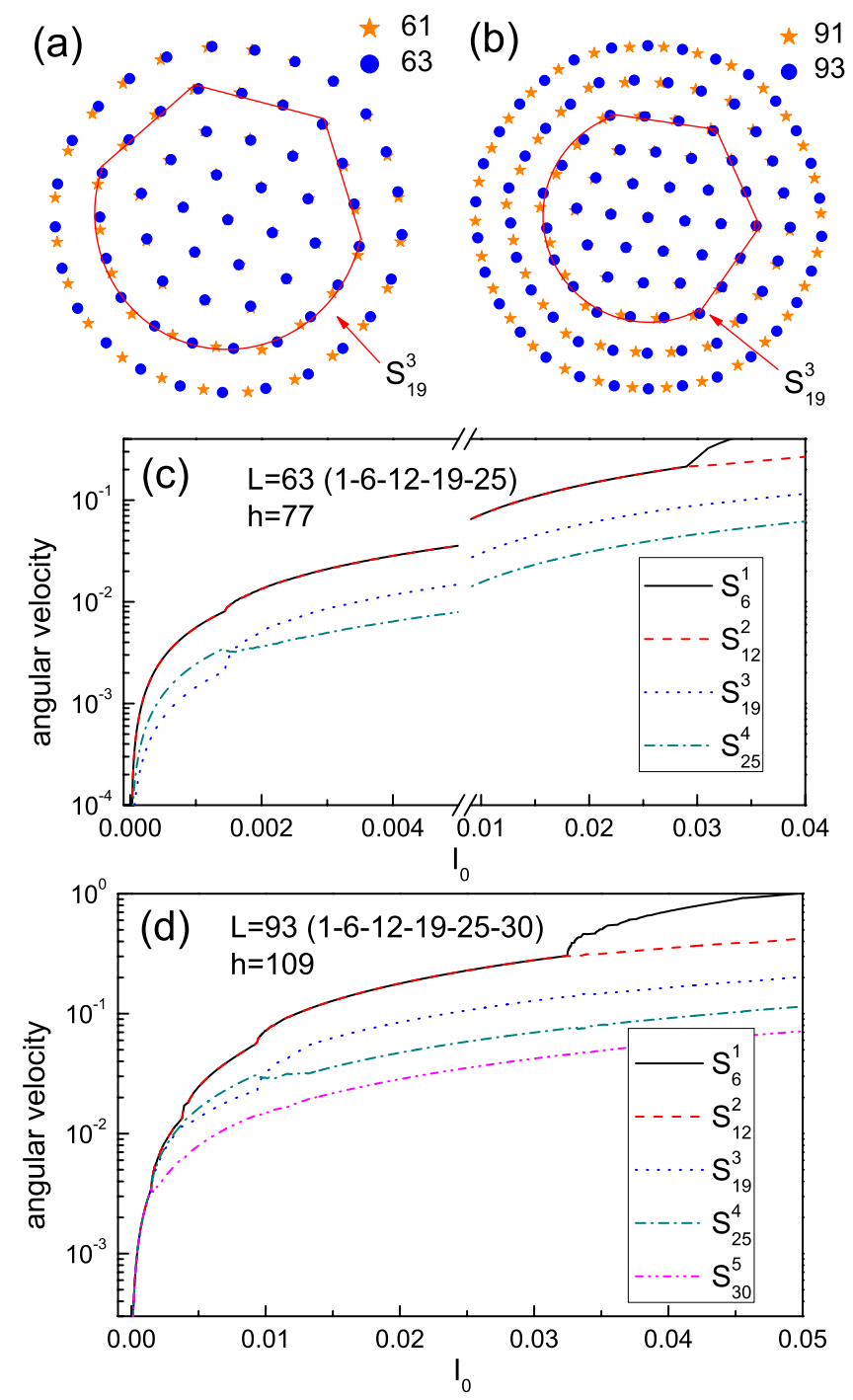

FIG. 7. (Color online) Comparison between the configurations: (a) (1-6-12-18-24) for $L=61$ and (1-6-12-19-25) for $L=63$; (b) (16-12-18-24-30) for $L=91$ and (1-6-12-19-25-30) for $L=93$, and the angular velocities of shells with (c) (1-6-12-19-25) for $L=63$, and (d) (1-6-12-19-25-30) for $L=93$. As shown here, the angular velocity of shell $S_{19}^{3}$ is smaller than that of shell $S_{25}^{4}$ when $I_{0}<0.0015$ (c) and when $0.005<I_{0}<0.009$ (d). However, shell $S_{19}^{3}$ experiences a larger Lorentz force than shell $S_{25}^{4}$ in both cases. Thus, the angular velocities of shells $S_{19}^{3}$ and $S_{25}^{4}$ are opposite to the gradient of the Lorentz force in these regimes, i.e., there is an inversion of the velocity of these two shells with respect to the usual case.

$$
v_{i}=\omega_{i} r_{i}=\omega_{c} r_{i}+\Delta v_{i} \quad(i=4,5),
$$

where $\omega_{c}$ is the angular velocity of the core, $r_{i}$ is the radius of the $i$ th shell, and $\Delta v$ is the linear velocity of the compression-decompression wave. The same linear velocity $\left(\Delta v_{4}=\Delta v_{5}\right)$ was found for shells $S_{25}^{4}$ and $S_{31}^{5}$ in the regime of compression-decompression motion, which become different for $I>I_{c}^{*}$ (shown in the inset of Fig. 9). Even in the case of several defect shells and for defect shells which are near the center of the disk, we find that the vortices of defect shells have the same $\Delta v$ in that regime (e.g., $L=94$ is shown in Fig. 


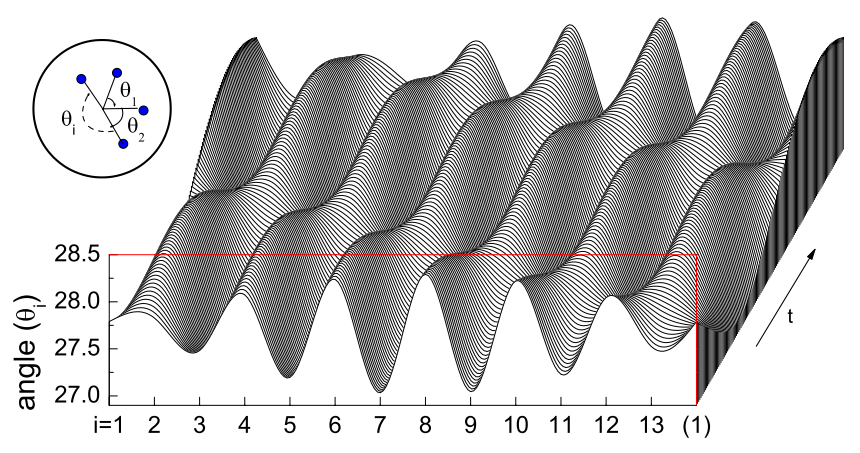

FIG. 8. (Color online) The evolution of the angle $\left(\theta_{i}\right)$ between two neighbor vortices in the incommensurate shell. The plot shows that the compression part (i.e., characterized by a relatively smaller angle $\theta_{i}$ ) propagates along the shell. The compression part is followed by the decompression part resulting in a propagating compression-decompression wave.

10). The compression-decompression wave of those defective shells $\left(S_{25}^{4}, S_{31}^{5}\right)$ have the same $\Delta v$. As seen from Fig. 11, the configuration shown by filled blue (dark gray) circles and the empty yellow (light gray) stars form the same configuration (1-6-12-18-25-31) rotated by $180^{\circ}$. The vortices of the two outer shells have to move only a small distance with respect to the core in order to restore the same initial configuration after rotation. The distance between two adjacent vortices $d_{0}$ is nearly the same and each vortex of the shells $S_{25}^{4}$ and $S_{31}^{5}$ has to slide over $d_{0}$ when the symmetry axis is rotated over $360^{\circ}$. Hence, we obtain nearly the same relative linear velocities of the shells until the current becomes large enough to make the shells move individually.

\section{Intershell defect and hysteresis}

We investigated also a very different configuration with several intershell vortices, for instance, $L=89(1-6-12-18$

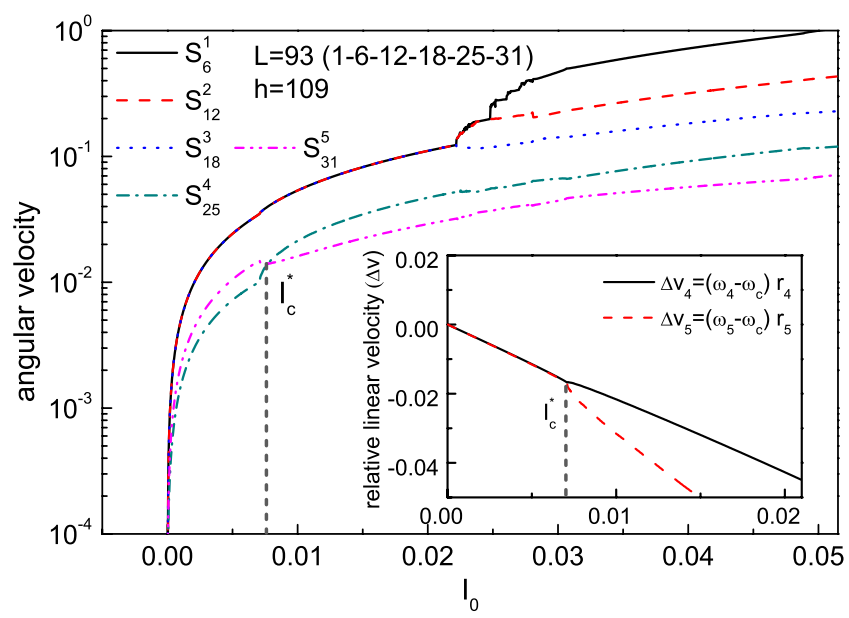

FIG. 9. (Color online) The evolution of angular velocities of shells with $L=93$ (1-6-12-18-25-31). The inset shows the relative linear velocities of $S_{25}^{4}$ and $S_{31}^{5}$ (i.e., the linear velocities of the relative backward motion with respect to the core part of the vortex configuration). The relative linear velocities are nearly the same until the current reaches the critical value $I_{c}^{*}$.

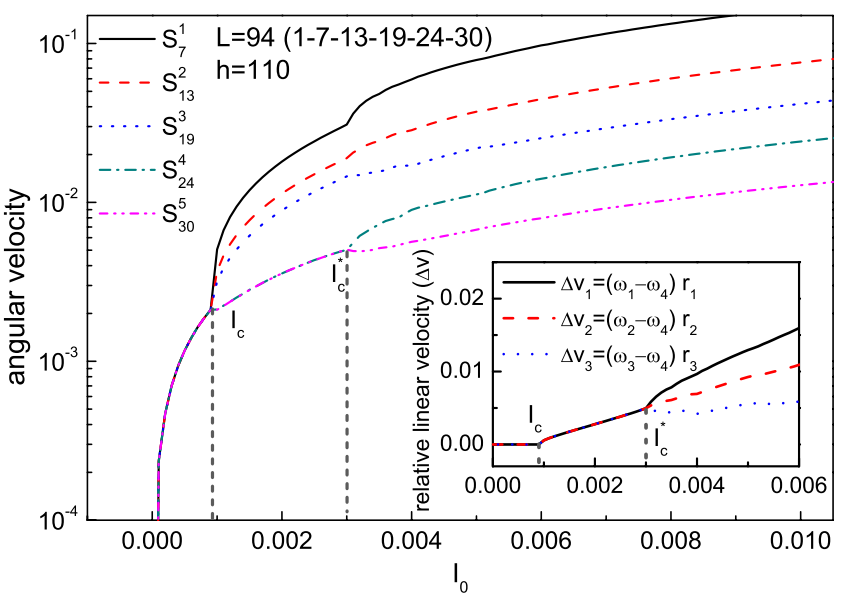

FIG. 10. (Color online) The evolution of angular velocities of shells with $L=94$ (1-7-13-19-24-30). The inset shows that the relative linear velocities of $S_{7}^{1}, S_{13}^{2}$, and $S_{19}^{3}$ with respect to the outer shells are the same until the outer shells unlock.

$=23=29$ ) (see inset of Fig. 12). Here, we have another kind of defect, called "intershell defect," which always locks three or more shells, e.g., shells $S_{18}^{3}, S_{23}^{4}$, and $S_{29}^{5}$ in configuration $(1-6-12-18=23=29)$ are locked until $I_{0}>0.014$, where the locking of shells is represented by the symbol " $=$ " instead of "-." Once unlocked, all the shells rotate separately under the action of the applied current.

The configuration $L=89$ in the absence of any applied current is shown in the inset of Fig. 12. It is nearly sixfold symmetric except for the outermost shell which is almost a circle due to the effect of the boundary. However, vortex rows become aligned along a straight line [shown by the blue (dark gray) lines in the inset of Fig. 12], i.e., along one of the symmetry axes. One of these rows is longer than the others and locks the three outer shells for $I_{0}<I_{c}$ and all the vortices rotate as a rigid body [shown in Fig. 13(a)]. For $I_{0}>I_{c}$, vortex "D" (shown in the inset of Fig. 12) jumps to the middle shell $\left(S_{17+D}^{3}\right)$ and the outermost vortex next to it is pushed

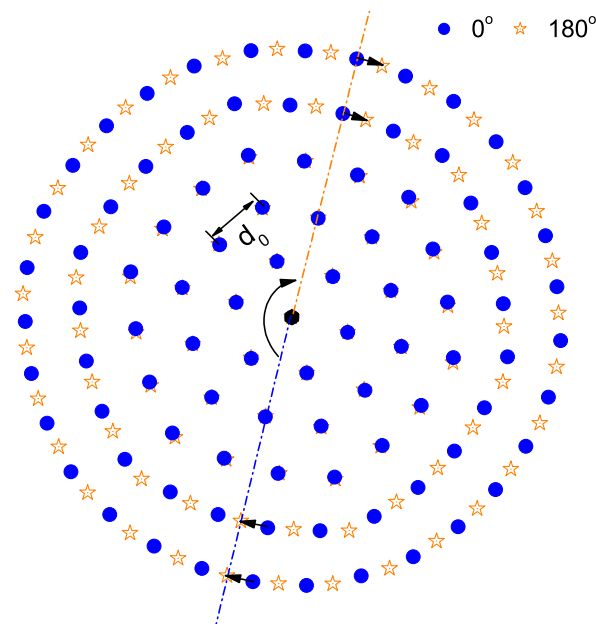

FIG. 11. (Color online) The filled blue (gray) circles and the empty yellow (light gray) stars show the locations of vortices before and after rotation over $180^{\circ}$ for $L=93$ (1-6-12-18-25-31). 


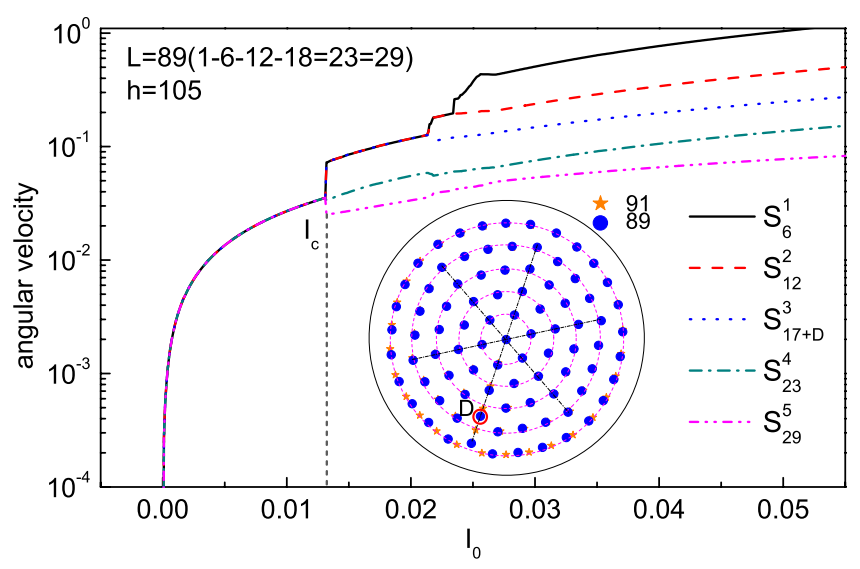

FIG. 12. (Color online) The average angular velocities of shells versus driving current $I_{0}$. When $I_{0}>I_{c}$, the intershell vortex $\mathrm{D}$ jumps into the middle shell $\left(S_{17}^{3}\right)$.

into the outermost shell $\left(S_{29}^{5}\right)$. These opposite radial movements of vortices in the row with a defect [Fig. 13(b)] finally rearrange the vortices into a well-defined shell structure (i.e., "dynamical ordering"). Although the gradient of the Lorentz force in the central part of the disk is not large enough to break the rigid-body motion of the core (1-6-12), it is sufficient to overcome the friction among all of the locked shells $S_{17+D}^{3}, S_{23}^{4}$, and $S_{29}^{5}$.

For the configuration (1-6-12-17=23=29) with $L=88$, we find that another intershell defect appears along another symmetric axis [the blue (dark gray) line in Fig. 14(a)]. With increasing driving force, vortex "D1" moves to the middle shell $\left(S_{16}^{3}\right)$ while vortex "D2" moves to the second outer shell $\left(S_{22}^{4}\right)$ [Fig. 14(b)]. Finally, the intershell defects disappear and all the vortices are arranged in the five-shell structure $\left(S_{6}^{1}, S_{12}^{2}, S_{17}^{3}, S_{23}^{4}, S_{29}^{5}\right)$ when $I_{0}>I_{c}$. The driving force then is large enough to unlock all the locked shells $S_{17}^{3}, S_{23}^{4}$, and $S_{29}^{5}$, however, the core (1-6-12) is still stable and rotates as a rigid body.

For $I_{0}<I_{c}$, the driving current is unable to destroy the radial vortex line(s) with defects. All the vortices rotate in the rigid-body mode. When the vortex system has transited from the static stable state to the dynamic steady state (with a well-defined shell structure), the system will not collapse into the static stable state immediately if we slightly decrease (a)

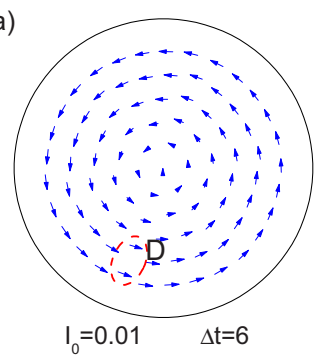

(b)

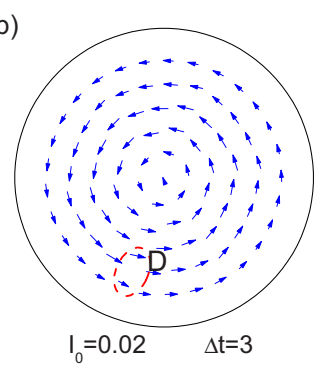

FIG. 13. (Color online) The vortex displacements with $L=89$ for: (a) driving current $I_{0}=0.01$, total time $\Delta t=6$ (6000 MD steps), and (b) driving current $I_{0}=0.02$, total time $\Delta t=3$. When $I_{0}<I_{c}$, the intershell vortex D stays between two shells $\left(S_{17}^{3}\right.$ and $\left.S_{23}^{4}\right)$. When $I_{0}>I_{c}$, the intershell vortex D jumps to the middle shell $S_{17}^{3}$.
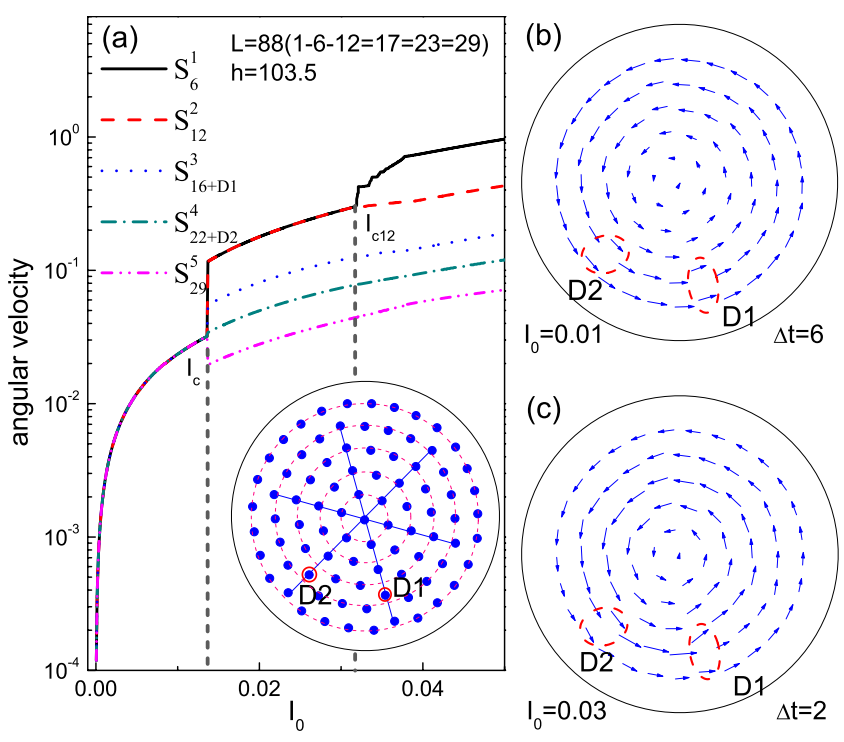

FIG. 14. (Color online) (a) The configuration and the average angular velocities of the different shells versus driving current $I_{0}$. The vortex displacements with $L=88$ for: (b) driving current $I_{0}$ $=0.01$, total time $\Delta t=6(6000 \mathrm{MD}$ steps $)$ and (c) driving current $I_{0}=0.03$, total time $\Delta t=2$. The defects are marked by the small dashed rings. When $I_{0}>I_{c}$, the intershell vortex "D1" jumps to the middle shell $S_{16}^{3}$ and the intershell vortex "D2" jumps to the shell $S_{22}^{4}$.

$I_{0}$ below $I_{c}$ (see Fig. 15). However, with further decreasing $I_{0}$ (i.e., $I_{0}<I_{c}^{-}$), the dynamic state becomes unstable and the system returns to the static stable state, thus all the vortices have the same angular velocity. The critical current $\left(I_{c}\right)$ to unlock $S_{16+D 1}^{3}$ and $S_{22+D 2}^{4}$ with increasing current is much larger than the critical current $\left(I_{c}^{-}\right)$when the system returns to

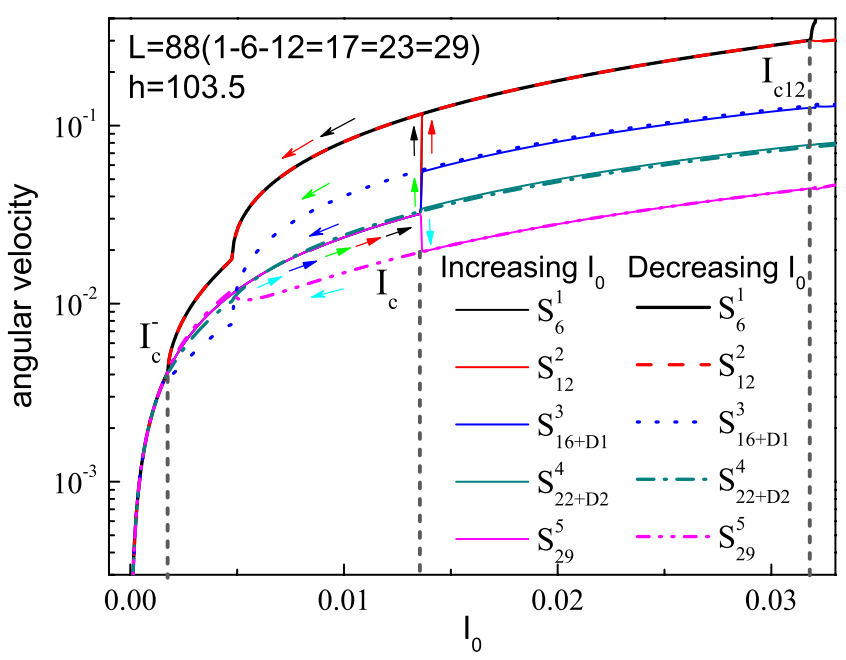

FIG. 15. (Color online) The angular velocities of the different shells when the driving current is increased (solid lines) or decreased (dashed lines of the same colors). $I_{c}$ is the critical value for breaking the rigid-body motion by increasing current while $I_{c}^{-}$is the critical current for returning to the rigid-body motion when decreasing current. When the current is between $I_{c}$ and $I_{c}^{-}$, the angular velocity of each shell is different when increasing and decreasing current (i.e., the hysteresis effect of angular velocity). 
the rigid-body rotation with decreasing current. In this regime, for the same value of the applied current, we can have different distributions of angular velocities, i.e., hysteresis in angular melting is found. The $I_{c 12}$ is the same for increased or decreased current (as shown in Fig. 15), therefore, there is no hysteresis behavior except for the shells which are locked by intershell defect(s). Namely, intershell defects are responsible for the hysteresis effect in the angular velocity.

\section{CONCLUSIONS}

The process of angular melting was investigated in a multishell (typically, three to five shells) system. Due to the interplay between the (intrashell and intershell) vortex-vortex interaction, the (in)commensurability between the shells, the effect of the boundary, and the gradient in the Lorentz force the process of angular melting becomes very complex in large mesoscopic disks. First, different scenarios of angular melting were found for different configurations. By analyzing the average angular velocity of the vortices in each shell, we found that either an inner shell or an outer shell can first unlock (i.e., the shell starts to rotate with different angular velocity with respect to the other shells); the former represents an usual ("classical") scenario of angular melting, the latter is referred to as "unconventional" angular melting. Because of a larger friction between two commensurate shells than between incommensurate shells, the commensurability plays an important role in the angular-melting process. Then, besides all-commensurate-shell configurations (i.e., the magic-number configurations), we investigated two kinds of imperfect-sixfold systems, which include intrashell defect(s) and intershell defect(s), respectively. An intrashell defect can cause an inversion of the angular velocities of the shells, i.e., when a shell which is closer to the center and thus experiences a larger Lorentz force moves slower than the adjacent shell with larger radius. To understand this unusual behavior, we distinguished three phases in the angular-melting process. The above unconventional dynamics is observed only in one of those phases, namely, during the compressiondecompression motion. When an extra (defect) vortex is present that shifts the other vortices from their triangularlattice positions, a part of the shell becomes nearly circlelike whereas another part keeps the hexagonal shape. In this case, the local shear modulus becomes angle dependent. Therefore, when the shear stress is not large enough to unlock the whole defect shell, the shell is partly melted and induces a very particular dynamical behavior, i.e., the motion in the form of a propagating compression-decompression wave. An intershell defect always locks several shells which rotate together up to some critical current. When the shear stress finally breaks the radial vortex line(s) containing defect(s), all shells that were locked by the defect release and start rotating individually with different angular velocities. Furthermore, such a lock-unlock process causes a hysteresis effect in the angular velocities of the shells. Therefore, different angular velocities of vortex shells have been found depending on the history, i.e., whether the applied current is increased or decreased.

\section{ACKNOWLEDGMENTS}

This work was supported by the "Odysseus" program of the Flemish Government and the Flemish Science Foundation (FWO-Vl), the Interuniversity Attraction Poles (IAP) Programme-Belgian State-Belgian Science Policy, and the FWO-Vl.
${ }^{1}$ S. F. W. R. Rycroft, R. A. Doyle, D. T. Fuchs, E. Zeldov, R. J. Drost, P. H. Kes, T. Tamegai, S. Ooi, and D. T. Foord, Phys. Rev. B 60, R757 (1999).

${ }^{2}$ Y. Paltiel, E. Zeldov, Y. Myasoedov, M. L. Rappaport, G. Jung, S. Bhattacharya, M. J. Higgins, Z. L. Xiao, E. Y. Andrei, P. L. Gammel, and D. J. Bishop, Phys. Rev. Lett. 85, 3712 (2000).

${ }^{3}$ A. A. Babaei Brojeny and J. R. Clem, Phys. Rev. B 64, 184507 (2001).

${ }^{4}$ D. López, W. K. Kwok, H. Safar, R. J. Olsson, A. M. Petrean, L. Paulius, and G. W. Crabtree, Phys. Rev. Lett. 82, 1277 (1999).

${ }^{5}$ G. W. Crabtree, D. López, W. K. Kwok, H. Safar, and L. M. Paulius, J. Low Temp. Phys. 117, 1313 (1999).

${ }^{6}$ M. C. Miguel and S. Zapperi, Nature Mater. 2, 477 (2003).

${ }^{7}$ V. R. Misko and F. M. Peeters, Phys. Rev. B 74, 174507 (2006).

${ }^{8}$ P. Benetatos and M. C. Marchetti, Phys. Rev. B 65, 134517 (2002).

${ }^{9}$ A. A. Abrikosov, Zh. Eksp. Teor. Fiz. 32, 1442 (1957) [Sov. Phys. JETP 5, 1174 (1957)].

${ }^{10}$ L. R. E. Cabral, B. J. Baelus, and F. M. Peeters, Phys. Rev. B 70, 144523 (2004).

${ }^{11}$ B. J. Baelus, L. R. E. Cabral, and F. M. Peeters, Phys. Rev. B 69 , 064506 (2004).

${ }^{12}$ V. R. Misko, B. Xu, and F. M. Peeters, Phys. Rev. B 76, 024516
(2007).

${ }^{13}$ I. V. Grigorieva, W. Escoffier, J. Richardson, L. Y. Vinnikov, S. Dubonos, and V. Oboznov, Phys. Rev. Lett. 96, 077005 (2006).

${ }^{14}$ I. V. Grigorieva, W. Escoffier, V. R. Misko, B. J. Baelus, F. M. Peeters, L. Y. Vinnikov, and S. V. Dubonos, Phys. Rev. Lett. 99, 147003 (2007).

${ }^{15}$ V. M. Bedanov and F. M. Peeters, Phys. Rev. B 49, 2667 (1994).

${ }^{16}$ S. W. S. Apolinario, B. Partoens, and F. M. Peeters, Phys. Rev. E 74, 031107 (2006).

${ }^{17}$ K. Nelissen, B. Partoens, I. Schweigert, and F. M. Peeters, Europhys. Lett. 74, 1046 (2006).

${ }^{18}$ N. S. Lin, V. R. Misko, and F. M. Peeters, Phys. Rev. Lett. 102, 197003 (2009)

${ }^{19}$ Following the previous works, we use term "angular melting" for consequent unlocking of vortex shells to distinguish it from "radial melting" (i.e., when vortices jump between shells and thus shells dissolve). This angular melting can be induced either by temperature (note that in this case the melting temperature of angular melting, $T_{a m}$, can be much lower than that of radial melting, $\left.T_{r m}: T_{a m} \ll T_{r m}\right)$ or by shear. In the latter case, it is similar to the "shear-induced melting transition" in colloidal crystals.

${ }^{20}$ C. Reichhardt, C. J. Olson, and F. Nori, Phys. Rev. Lett. 78, 
2648 (1997); Phys. Rev. B 57, 7937 (1998); 58, 6534 (1998).

${ }^{21}$ V. R. Misko, S. Savel'ev, A. L. Rakhmanov, and F. Nori, Phys. Rev. Lett. 96, 127004 (2006); Phys. Rev. B 75, 024509 (2007).

${ }^{22}$ W. V. Pogosov, V. R. Misko, H. J. Zhao, and F. M. Peeters, Phys. Rev. B 79, 014504 (2009); W. V. Pogosov, H. J. Zhao, V. R. Misko, and F. M. Peeters, ibid. 81, 024513 (2010).

${ }^{23}$ A. I. Buzdin and J. P. Brison, Phys. Lett. A 196, 267 (1994).

${ }^{24}$ We use the term ground state in this work to denote the classical state with the lowest free energy following the previous papers (e.g., see Refs. 25 and 26).

${ }^{25}$ P. A. Venegas and E. Sardella, Phys. Rev. B 58, 5789 (1998).

${ }^{26}$ V. A. Schweigert, F. M. Peeters, and P. S. Deo, Phys. Rev. Lett. 81, 2783 (1998).

${ }^{27}$ K. Harada, O. Kamimura, H. Kasai, T. Matsuda, A. Tonomura, and V. V. Moshchalkov, Science 274, 1167 (1996).

${ }^{28}$ T. Sasagawa, Y. Togawa, J. Shimoyama, A. Kapitulnik, K. Kitazawa, and K. Kishio, Phys. Rev. B 61, 1610 (2000).

${ }^{29}$ In our simulation, the radius of the disk is $50 \xi$ and the number of shells is not more than five. Therefore, roughly the distance between two vortices is larger than $8 \xi$, i.e., they are well separated.

${ }^{30}$ M. Kong, B. Partoens, and F. M. Peeters, Phys. Rev. E 65, 046602 (2002).

${ }^{31}$ V. A. Schweigert and F. M. Peeters, Phys. Rev. B 51, 7700 (1995).

${ }^{32}$ M. Kong, B. Partoens, and F. M. Peeters, Phys. Rev. E 67, 021608 (2003).

${ }^{33}$ L. J. Campbell and R. M. Ziff, Phys. Rev. B 20, 1886 (1979). 